PROCEEDINGS OF THE

AMERICAN MATHEMATICAL SOCIETY

Volume 129, Number 9, Pages 2809-2815

S 0002-9939(01)05883-X

Article electronically published on February 9, 2001

\title{
VIETORIS CONTINUOUS SELECTIONS AND DISCONNECTEDNESS-LIKE PROPERTIES
}

\author{
VALENTIN GUTEV AND TSUGUNORI NOGURA
}

(Communicated by Alan Dow)

\begin{abstract}
Suppose that $X$ is a Hausdorff space such that its Vietoris hyperspace $\left(\mathcal{F}(X), \tau_{V}\right)$ has a continuous selection. Do disconnectedness-like properties of $X$ depend on the variety of continuous selections for $\left(\mathcal{F}(X), \tau_{V}\right)$ and vice versa? In general, the answer is "yes" and, in some particular situations, we were also able to set proper characterizations.
\end{abstract}

\section{Introduction}

Let $X$ be a Hausdorff space, and let $\mathcal{F}(X)$ be the family of the non-empty closed subsets of $X$. Also, let $\mathcal{D} \subset \mathcal{F}(X)$. A map $f: \mathcal{D} \rightarrow X$ is a selection for $\mathcal{D}$ if $f(S) \in S$ for every $S \in \mathcal{D}$. A map $f: \mathcal{D} \rightarrow X$ is a continuous selection for $\mathcal{D}$ if it is a selection which is continuous with respect to the relative Vietoris topology $\tau_{V}$ on $\mathcal{D}$. Let us recall that the Vietoris topology $\tau_{V}$ on $\mathcal{F}(X)$ is generated by all collections of the form

$$
\langle\mathcal{V}\rangle=\{S \in \mathcal{F}(X): S \cap V \neq \emptyset, V \in \mathcal{V}, \text { and } S \subset \bigcup \mathcal{V}\}
$$

where $\mathcal{V}$ runs over the finite families of open subsets of $X$.

In what follows, we use $\mathcal{S e \ell}(X)$ to denote the set of all continuous selections for $\mathcal{F}(X)$, and $\operatorname{dim}(X)$ to denote the covering dimension of $X$. Also, we denote by $\operatorname{ind}(X)$ the small inductive dimension of $X$. Finally, we shall say that $X$ is zerodimensional if it has a base of clopen sets (i.e., if ind $(X)=0$ ), and that $X$ is strongly zero-dimensional if $\operatorname{dim}(X)=0$. Note that every strongly zero-dimensional space is zero-dimensional but the converse fails ([12], [13], see also [11]).

The dimension-type of restrictions play an important role in the selection theory for hyperspaces. For instance, $\operatorname{Se\ell }(X) \neq \emptyset$ for every strongly zero-dimensional completely metrizable space $X$ (see [3], [5]). On the other hand, we have the following two results in the opposite direction.

Theorem 1.1 ([8]). If $X$ is a compact Hausdorff space with $\mathcal{S e \ell}(X) \neq \emptyset$, then it is a linear ordered topological space. In particular, $\operatorname{dim}(X) \leq 1$.

Theorem $1.2([10])$. If $X$ is a compact Hausdorff space with $\operatorname{Se\ell }(X) \neq \emptyset$, then it has finitely many connected components if and only if $\mathcal{S e \ell}(X)$ is finite. In particular, $\operatorname{dim}(X)=1$ provided $X$ is infinite and $\mathcal{S e \ell}(X)$ is finite.

Received by the editors November 17, 1999 and, in revised form, January 17, 2000.

2000 Mathematics Subject Classification. Primary 54C65, 54B20, 54F45.

Key words and phrases. Selections, hyperspaces, zero-dimensionality.

(C)2001 American Mathematical Society 
In the present paper we are interested in relations between the set $\operatorname{Sel}(X)$ and zero-dimensionality of $X$. As Theorems 1.1 and 1.2 suggest, we may expect "sufficiently many" continuous selections for $\mathcal{F}(X)$ provided $\operatorname{dim}(X)=0$ and $\operatorname{Se} \ell(X) \neq \emptyset$. In fact, this is the first result of the paper. The following theorem will be proved in the next section.

Theorem 1.3. If $X$ is a zero-dimensional Hausdorff space such that $\operatorname{Se\ell }(X) \neq \emptyset$, then the set $\{f(X): f \in \mathcal{S e \ell}(X)\}$ is dense in $X$.

The proof of Theorem 1.3 does not involve such complicated arguments. However, in general, the conclusion " $\{f(X): f \in \mathcal{S} e \ell(X)\}$ is dense in $X$ " cannot be strengthened to " $\{f(X): f \in \mathcal{S e \ell}(X)\}=X$ " (see Example 2.1). On the other hand, this becomes possible provided $X$ is a first countable space which allows us to obtain also the converse.

Theorem 1.4. Let $X$ be a first countable Hausdorff space such that $\mathcal{S} e \ell(X) \neq \emptyset$. Then it is zero-dimensional if and only if for every point $x \in X$ there exists $f_{x} \in$ $\operatorname{Se\ell }(X)$ such that $f_{x}^{-1}(x)=\{S \in \mathcal{F}(X): x \in S\}$.

In view of Theorem 1.4, it would be interesting to know if the converse of Theorem 1.3 holds as well. Here we have only the following partial result.

Theorem 1.5. If $X$ is a Hausdorff space such that $\{f(X): f \in \mathcal{S e l}(X)\}$ is dense in $X$, then it is totally disconnected.

Since every totally disconnected locally compact space is zero-dimensional, by Theorems 1.3 and 1.5, we get the following consequence.

Corollary 1.6. Let $X$ be a locally compact Hausdorff space such that $\operatorname{Se\ell }(X) \neq \emptyset$. Then $\operatorname{ind}(X)=0$ if and only if $\{f(X): f \in \mathcal{S e \ell}(X)\}$ is dense in $X$.

A word should be said about the proofs of Theorems 1.4 and 1.5. A preparation for that is done in Sections 3 and 4. A proof of Theorem 1.5 is obtained in Section 5. Since the proof of Theorem 1.4 involves that of Theorem 1.5, it will be finally accomplished in Section 6 of the paper.

\section{SeleCtions AND ClOpen SETS}

Throughout this section, and in the sequel, $X$ is always a Hausdorff space. We first prove Theorem 1.3. In fact, this theorem is a consequence of the following lemma.

Lemma 2.1. Let $X$ be a space such $\mathcal{S e \ell}(X) \neq \emptyset$, and let $G$ be a non-empty clopen subset of $X$. Then there exists a selection $g \in \operatorname{Se\ell }(X)$ with $g(X) \in G$.

Proof. Note that the sets

$$
\mathcal{G}_{0}=\{S \in \mathcal{F}(X): S \cap G=\emptyset\} \quad \text { and } \quad \mathcal{G}_{1}=\{S \in \mathcal{F}(X): S \cap G \neq \emptyset\}
$$

constitute a disjoint and $\tau_{V}$-open cover of $\mathcal{F}(X)$. Define another subset $\mathcal{G}_{2}$ of $\mathcal{F}(X)$ by $\mathcal{G}_{2}=\{S \in \mathcal{F}(X): S \subset G\}$. Take a selection $f \in \mathcal{S e \ell}(X)$. Note that each $f_{i}=f \mid \mathcal{G}_{i}, i=0,1,2$, is a continuous selection for $\mathcal{G}_{i}$. We now consider the map $\varphi: \mathcal{G}_{1} \rightarrow \mathcal{G}_{2}$ defined by $\varphi(S)=S \cap G$ for every $S \in \mathcal{G}_{1}$. This map is continuous with respect to the relative Vietoris topologies on $\mathcal{G}_{1}$ and $\mathcal{G}_{2}$. Finally, define a map $g: \mathcal{F}(X) \rightarrow X$ by $g \mid \mathcal{G}_{0}=f_{0}$ and $g \mid \mathcal{G}_{1}=f_{2} \circ \varphi$. Clearly, $g$ is a selection for $\mathcal{F}(X)$. That $g \in \operatorname{Se\ell }(X)$ follows from the fact that $\varphi$ is continuous and $\mathcal{G}_{0}$ and $\mathcal{G}_{1}$ 
are $\tau_{V}$-open in $\mathcal{F}(X)$. This $g$ is the required one because $X \in \mathcal{G}_{1}$ and, therefore, $g(X)=f_{2}(\varphi(X))=f_{2}(X \cap G)=f(G) \in G$.

Example 2.2. There exists a space $X_{p}$ with only one non-isolated point $p \in X_{p}$ such that $\operatorname{Se\ell }\left(X_{p}\right) \neq \emptyset$ and $f\left(X_{p}\right) \neq p$ for every $f \in \mathcal{S} e \ell\left(X_{p}\right)$.

Proof. Let $p=\omega_{1}$ be the first uncountable ordinal, and let $X_{p}=\{\alpha+1: \alpha<$ $\left.\omega_{1}\right\} \cup\{p\}$. We endow $X_{p}$ with the relative topology as a subspace of the ordinal space $\omega_{1}+1$. Then, $X_{p}$ is a space with only one non-isolated point $p$ such that every countable subset of $X_{p} \backslash\{p\}$ is closed in $X_{p}$. Hence, by a result of [1], $f\left(X_{p}\right) \neq p$ for every $f \in \operatorname{Se\ell }\left(X_{p}\right)$. On the other hand, $X_{p}$ is a linear ordered space and $f(S)=\min S, S \in \mathcal{F}\left(X_{p}\right)$, defines a continuous selection for $\mathcal{F}\left(X_{p}\right)$.

In contrast to Example 2.2, we have the following lemma demonstrating the relationship of the problem with the convergent structure of $X_{p}$ in $p$.

In what follows, we use $\omega$ to denote the first infinite ordinal. As a space, every ordinal has the usual order topology.

Lemma 2.3. Let $X$ be a space, $p \in X$, and let $Y_{q}$ be the quotient space on the disjoint union $X \sqcup(\omega+1)$ obtained by identifying the points $p$ and $\omega$ to a single point $q \in Y_{q}$. If $\operatorname{Se\ell }\left(Y_{q}\right) \neq \emptyset$, then there exists $g \in \operatorname{Se\ell }\left(Y_{q}\right)$ with $g\left(Y_{q}\right)=q$.

Proof. Let

$$
\mathcal{V}_{0}=\left\{F \in \mathcal{F}\left(Y_{q}\right): 0 \notin F\right\} \quad \text { and } \quad \mathcal{V}_{1}=\left\{F \in \mathcal{F}\left(Y_{q}\right): 0 \in F\right\} .
$$

Since $0 \in Y_{q}$ is an isolated point, this defines a disjoint $\tau_{V}$-open cover $\left\{\mathcal{V}_{0}, \mathcal{V}_{1}\right\}$ of $\mathcal{F}\left(Y_{q}\right)$. Consider the sets

$$
\mathcal{V}_{1}^{0}=\left\{F \in \mathcal{V}_{1}: \omega \backslash F \neq \emptyset\right\} \quad \text { and } \quad \mathcal{V}_{1}^{1}=\left\{F \in \mathcal{V}_{1}: \omega \subset F\right\} .
$$

Next, for every $F \in \mathcal{V}_{1}^{0}$, define $n(F)=\min \{n \in \omega \cap F: n+1 \notin F\}$. Now, take $f \in \mathcal{S e \ell}\left(Y_{q}\right)$ and then define another selection $g: \mathcal{F}\left(Y_{q}\right) \rightarrow Y_{q}$ for $\mathcal{F}\left(Y_{q}\right)$ by $g \mid \mathcal{V}_{0}=$ $f \mid \mathcal{V}_{0}$ while $g(F)=n(F)$ if $F \in \mathcal{V}_{1}^{0}$ and $g(F)=q$ otherwise. Since $Y_{q} \in \mathcal{V}_{1}^{1}$, we have $g\left(Y_{q}\right)=q$. Thus to finish the proof it only remains to show that $g$ is continuous. In fact, it suffices to show that $g \mid \mathcal{V}_{1}$ is continuous because $g\left|\mathcal{V}_{0}=f\right| \mathcal{V}_{0}$. Take an $F \in \mathcal{V}_{1}$. We distinguish the following two cases. If $F \in \mathcal{V}_{1}^{0}$, then $g(F)=n(F)<\omega$ and $\{n<\omega: n \leq n(F)\} \subset F$. Set $\mathcal{U}=\{\{n\}: n \leq n(F)\} \cup\left\{Y_{q} \backslash\{n(F)+1\}\right\}$. Then $\langle\mathcal{U}\rangle \subset \mathcal{V}_{1}^{0}$ is a $\tau_{V}$-neighbourhood of $F$ such that $n(S)=n(F)$ for every $S \in\langle\mathcal{U}\rangle$. Hence, $g(\langle\mathcal{U}\rangle)=\{g(F)\}$. Suppose now that $F \in \mathcal{V}_{1}^{1}$, and let $V$ be a neighbourhood of $q=g(F)$. Take an $m<\omega$ such that $\{n<\omega: n \geq m\} \subset V$. Then, let $\mathcal{U}=\{\{n\}: n \leq m\} \cup\left\{Y_{q}\right\}$. In this way, we get a $\tau_{V}$-neighbourhood $\langle\mathcal{U}\rangle$ of $F$ such that $g(\langle\mathcal{U}\rangle) \subset V$. Indeed, take an $S \in\langle\mathcal{U}\rangle$. In case $\omega \subset S$, by definition, we have $g(S)=q \in V$. Otherwise, $\{n \in \omega \cap S: n \leq m\} \subset S$ implies that $n(S) \geq m$. Therefore, $g(S)=n(S) \in V$.

\section{SELECTIONS AND ORDER-LIKE RELATIONS}

In this section we collect some known facts we need for the proof of Theorem 1.5. Suppose that $X$ is a space with $\operatorname{Se\ell }(X) \neq \emptyset$. Following 7], to every selection $f \in \mathcal{S e} \ell(X)$ we associate an order-like relation $\preceq_{f}$ on $X$ defined for $x, y \in X$ by

$$
x \preceq_{f} y \quad \text { if and only if } \quad f(\{x, y\})=x .
$$

In what follows, we shall refer to " $\preceq f$ " as an $f$-order on $X$. Also, let us agree to write $x \prec_{f} y$ provided $x \preceq_{f} y$ and $x \neq y$. 
Finally, for every $f \in \mathcal{S e} \ell(X)$ and $x \in X$, we consider the following special subsets of $X$ :

$$
\begin{aligned}
& (-\infty, x]_{f}=\left\{z \in X: z \preceq_{f} x\right\} \quad \text { and } \quad[x,+\infty)_{f}=\left\{z \in X: x \preceq_{f} z\right\}, \\
& (-\infty, x)_{f}=\left\{z \in X: z \prec_{f} x\right\} \quad \text { and } \quad(x,+\infty)_{f}=\left\{z \in X: x \prec_{f} z\right\} .
\end{aligned}
$$

The following observation is an immediate consequence of the continuity of $f$ (see 7. Lemma 7.2]) and we left the corresponding arguments to the interested reader.

Lemma 3.1. Let $X$ be a space, and let $f \in \operatorname{Se\ell }(X)$. Then, for every $x \in X$, the sets $(-\infty, x)_{f}$ and $(x,+\infty)_{f}$ are open in $X$. In particular, $(-\infty, x]_{f}$ and $[x,+\infty)_{f}$ are closed in $X$.

It should be mentioned that, in contrast to usual linear orders on $X$, the $f$-orders are not engaged to be transitive. However, in case of connected spaces, this is so. The following result is a partial case of [7, Lemmas 7.2 and 7.3] (see also [9, Lemma 10]).

Lemma 3.2. Let $X$ be a connected space such that $\operatorname{Se\ell }(X) \neq \emptyset$. Then, $|\operatorname{Se\ell }(X)| \leq$ 2 and, for every $f \in \mathcal{S} e \ell(X)$, the following hold.

(1) The $f$-order on $X$ is transitive.

(2) $f(X)=\min _{\preceq_{f}} X$.

(3) $g(X)=\max _{\preceq_{f}} X$ provided $g \in \mathcal{S e \ell}(X) \backslash\{f\}$.

In case of connected subsets of $X$ we also have the following property of $f$-orders.

Lemma 3.3. Let $X$ be a space, $f \in \mathcal{S} e \ell(X)$, and let $A \subset X$ be connected. Also, let $x, y \in A$ be such that $x \preceq_{f} y$. Then,

$$
(x, y)_{f}=\left\{z \in X: x \prec_{f} z \prec_{f} y\right\} \subset A .
$$

Proof. Suppose that there exists $z \in(x, y)_{f} \backslash A$. Then, according to Lemma 3.1, $U=(-\infty, z]_{f} \cap A$ is a clopen (in $A$ ) subset of $A$ because $U=(-\infty, z)_{f} \cap A$. Note that $x \in U$ while $y \in A \backslash U$. Since $A$ is connected, this is impossible.

\section{Selections And components}

Let $X$ be a space. For every $x \in X$, we shall use $\mathcal{C}[x]$ to denote the component of the point $x$ and $\mathcal{C}^{*}[x]$ the corresponding quasi-component. Let us recall that

$$
\mathcal{C}[x]=\bigcup\{C \subset X: x \in C \text { and } C \text { is connected }\}
$$

and, respectively,

$$
\mathcal{C}^{*}[x]=\bigcap\{C \subset X: x \in C \text { and } C \text { is clopen }\} .
$$

In this section we establish the following result which may have some independent interest.

Theorem 4.1. Let $X$ be a space with $\operatorname{Se\ell }(X) \neq \emptyset$. Then, $\mathcal{C}^{*}[x]=\mathcal{C}[x]$ for every point $x \in X$.

To prepare for the proof of Theorem 4.1, we need the following lemma.

Lemma 4.2. Let $X$ be such that $\operatorname{Se\ell }(X) \neq \emptyset$, and let $f \in \operatorname{Se\ell }(X)$. Then for every $x \in X$ and $y, z \in \mathcal{C}^{*}[x]$, with $y \preceq_{f} z$, we have

$$
[y, z]_{f}=\left\{t \in X: y \preceq_{f} t \preceq_{f} z\right\} \subset \mathcal{C}^{*}[x] .
$$


Proof. Suppose that there are points $y, z \in \mathcal{C}^{*}[x]$ and $t \in X \backslash \mathcal{C}^{*}[x]$ such that $y \prec_{f}$ $t \prec_{f} z$. Since $t \notin \mathcal{C}^{*}[x]$, there exists a clopen subset $V \subset X$ such that $\mathcal{C}^{*}[x] \subset V$ and $t \notin V$. Then, by Lemma 3.1, $U=(-\infty, t]_{f} \cap V$ defines a clopen (in $X$ ) neighbourhood of $y$ because $U=(-\infty, t)_{f} \cap V$. Note that $z \notin U$. However, this is impossible because $z \in \mathcal{C}^{*}[x]=\mathcal{C}^{*}[y] \subset U$. A contradiction.

Proof of Theorem 4.1. Let $x \in X$. It suffices to show that $\mathcal{C}^{*}[x]$ is connected. Towards this end, note that, by Lemma 4.2,

$$
\mathcal{C}^{*}[x]=\bigcup\left\{[y, z]_{f}: y, z \in \mathcal{C}^{*}[x], y \preceq_{f} z \text {, and } y \preceq_{f} x \preceq_{f} z\right\} .
$$

Hence, it will be sufficient to show that for every $y, z \in \mathcal{C}^{*}[x]$, with $y \preceq_{f} z$, the set $[y, z]_{f}$ is connected. Suppose to the contrary that $[y, z]_{f}$ is not connected for some points $y, z \in \mathcal{C}^{*}[x]$ with $y \preceq_{f} z$. Then, there exists a clopen (in $[y, z]_{f}$ ) neighbourhood $W \subset[y, z]_{f}$ of $z$ such that $[y, z]_{f} \backslash W \neq \emptyset$. Take a point $t \in[y, z]_{f} \backslash W$ and then set $T=W \cap[t, z]_{f}$. Thus, we get a clopen (in $[t, z]_{f}$ ) neighbourhood $T$ of $z$ such that $t \notin T$. Then, the set $G=T \cup[z,+\infty)_{f}$ is clopen in $X$. Indeed, according to Lemma 3.1, $G$ is closed in $X$ as a union of two closed subsets. To show that it is also open in $X$, note that there exists an open subset $E \subset(t,+\infty)_{f}$ such that $E \cap[t, z]_{f}=T$ because $t \notin T \subset[t, z]_{f} \subset[t,+\infty)_{f}$. Hence, by Lemma 3.1, the set $G$ is open in $X$ because $G=E \cup(z,+\infty)_{f}$. Thus, $G$ is clopen in $X$. This however is impossible because $t \notin G$ and $z \in G$, while $t, z \in \mathcal{C}^{*}[x]$. A contradiction.

\section{Proof of Theorem 1.5}

Let $X$ be such that the set $D=\{f(X): f \in \mathcal{S e \ell}(X)\}$ is dense in $X$. Suppose if possible that $X$ is not totally disconnected. Then, by Theorem 4.1, $X$ must contain an infinite closed connected set $A$. Let $B=\{g(A): g \in \operatorname{Sel}(A)\}$, and let $f \in \operatorname{Se\ell }(X)$. Note that, by Lemmas 3.2 and 3.3, there exists $a=\min \preceq_{f} A$. According to the same lemmas, we consider the subset $H=\bigcup\left\{(a, x)_{f}: x \in A\right\}$ of $A \backslash B$. Note that it is non-empty because $A$ is infinite while, by Lemma 3.1, it is open in $X$. Then, by hypothesis, there exists a selection $h \in \mathcal{S} e \ell(X)$ with $h(X) \in H$. Since $h$ is continuous, there now exists a finite open cover $\mathcal{U}$ of $X$ such that $h(\langle\mathcal{U}\rangle) \subset H$. Take a finite $F \subset X \backslash A$ so that $Z=F \cup A \in\langle\mathcal{U}\rangle$. Then, we have that $h(Z) \in H \subset A$. Set $k=h \mid \mathcal{F}(Z)$ and $\mathcal{A}=\{S \cup F: S \in \mathcal{F}(A)\}$. Note that $\psi: \mathcal{F}(A) \rightarrow \mathcal{A}$, defined by $\psi(S)=S \cup F, S \in \mathcal{F}(A)$, becomes a continuous onto map. Since $\mathcal{F}(A)$ is connected (because so is $A$; see [7, Theorem 4.10]), this implies that $\mathcal{A}$ is also connected. On the other hand, $k^{-1}(A)$ is a $\tau_{V}$-clopen subset of $\mathcal{F}(Z)$ because $A$ is clopen in $Z$. Also, $A \cup F=Z \in k^{-1}(A)$ which finally implies that $\mathcal{A} \cap k^{-1}(A) \neq \emptyset$. Thus, $\mathcal{A} \subset k^{-1}(A)$. We may now define a continuous selection $g: \mathcal{F}(A) \rightarrow A$ by letting $g=k \circ \psi$. However, this selection has the property that $g(A)=k(\psi(A))=k(A \cup F)=h(A \cup F) \in H \subset A \backslash B$. A contradiction.

\section{Proof of Theorem 1.4}

Let $X$ be as in Theorem 1.4, and let $\operatorname{ind}(X)=0$. Take an $f \in \operatorname{Se\ell }(X)$, a point $x \in X$, and a decreasing clopen base $\mathcal{U}=\left\{U_{n}: n<\omega\right\}$ of $x$ in $X$ such that $U_{0}=X$. For reasons of convenience, let $U_{\omega}=\{x\}$. Now, for every $n \leq \omega$ define a subset $\mathcal{F}_{n}=\left\{S \in \mathcal{F}(X): S \cap U_{n} \neq \emptyset\right\}$. Note that $\mathcal{F}_{\omega}=\bigcap\left\{\mathcal{F}_{n}: n<\omega\right\}$ because $\mathcal{U}$ is a base at $x$, while $\mathcal{F}_{0}=\mathcal{F}(X)$ because $U_{0}=X$. Then, define a map $\theta: \mathcal{F}(X) \rightarrow \omega+1$ by setting $\theta(S)=\max \left\{n \leq \omega: S \in \mathcal{F}_{n}\right\}$ for every $S \in \mathcal{F}(X)$. Also, for every $n \leq \omega$, define a map $\varphi_{n}: \mathcal{F}_{n} \rightarrow \mathcal{F}(X)$ by $\varphi_{n}(S)=S \cap U_{n}, S \in \mathcal{F}_{n}$. Finally, define 
a map $g: \mathcal{F}(X) \rightarrow X$ by $g(S)=f\left(\varphi_{\theta(S)}(S)\right)$ for every $S \in \mathcal{F}(X)$. Thus, we get a selection $g$ for $\mathcal{F}(X)$ such that $g^{-1}(x)=\mathcal{F}_{\omega}$. It only remains to show that $g$ is continuous. Clearly, $g$ is continuous at the singleton $\{x\}$. So, take an $F \in \mathcal{F}(X)$ with $F \neq\{x\}$. We distinguish the following two cases. If $g(F)=x$, then for every $n<\omega$ let $\mathcal{V}_{n}=\left\{U_{n}, X \backslash U_{n}\right\}$. In this case, $g\left(\left\langle\mathcal{V}_{n}\right\rangle\right) \subset U_{n}$. Indeed, let $S \in\left\langle\mathcal{V}_{n}\right\rangle$. Then, $S \cap U_{n} \neq \emptyset$ implies $\theta(S) \geq n$. Hence, $g(S)=f\left(\varphi_{\theta(S)}(S)\right) \in U_{\theta(S)} \subset U_{n}$ because $\mathcal{U}$ is decreasing. Therefore, $g$ is continuous at $F$ because $\mathcal{U}$ is a local base at $g(F)$ while the set $\left\{n<\omega: F \notin\left\langle\mathcal{V}_{n}\right\rangle\right\}$ is finite. Suppose now that $g(F) \neq x$. The definition of $g$ implies that $m=\theta(F)<\omega$. Then, set $\mathcal{W}=\left\{U_{m} \backslash U_{m+1}, X \backslash U_{m+1}\right\}$. We have that $F \in\langle\mathcal{W}\rangle$ because $F \cap U_{m+1}=\emptyset$. On the other hand, $S \in\langle\mathcal{W}\rangle$ implies that $\theta(S)=m$ because $\mathcal{U}$ is decreasing, $S \cap U_{m} \neq \emptyset$ and $S \cap U_{m+1}=\emptyset$. This finally implies that $g$ is continuous at $F$ because $\langle\mathcal{W}\rangle \subset \mathcal{F}_{m}$ and $\varphi_{m} \mid\langle\mathcal{W}\rangle$ is continuous.

Suppose now that, for every $x \in X$, there exists $f_{x} \in \mathcal{S e \ell}(X)$ such that $f_{x}^{-1}(x)=$ $\{S \in \mathcal{F}(X): x \in S\}$. Next, take a point $x \in X$ and then set $g=f_{x}$. Also, let $V$ be a neighbourhood of $x$ such that $F=X \backslash V$ is non-empty, i.e. $F \in \mathcal{F}(X)$. Then, by hypothesis, $g(F \cup\{x\})=x$ and $g(F) \neq x$. Hence, by Theorem 1.5, there exists a clopen set $U$ such that $g(F) \in U$ and $x \notin U$. In this way, we get a $\tau_{V^{-}}$ clopen neighbourhood $g^{-1}(U)$ of $F$ in $\mathcal{F}(X)$. Let $\mathcal{M} \subset g^{-1}(U)$ be a chain which is maximal with respect to the usual set-theoretical inclusion and $F \in \mathcal{M}$. Then, there exists $M=\max \mathcal{M}$ because $g^{-1}(U)$ is $\tau_{V}$-closed (see [2, [4, 6]). Indeed, it suffices to show that $M=\overline{\cup \mathcal{M}} \in g^{-1}(U)$. Take a basic $\tau_{V}$-neighbourhood $\langle\mathcal{W}\rangle$ of $M$. Then, for every $W \in \mathcal{W}$ there exists $M_{W} \in \mathcal{M}$ with $M_{W} \cap W \neq \emptyset$ because $(\bigcup \mathcal{M}) \cap W \neq \emptyset$. Then, $M_{\mathcal{W}}=\bigcup\left\{M_{W}: W \in \mathcal{W}\right\} \in\langle\mathcal{W}\rangle \cap \mathcal{M}$ because $\mathcal{M}$ is a chain. Hence, in particular, $\langle\mathcal{W}\rangle \cap g^{-1}(U) \neq \emptyset$ which finally implies that $M \in g^{-1}(U)$ because $g^{-1}(U)$ is $\tau_{V}$-closed. Having established this, let us also observe that $M$ is open because $g^{-1}(U)$ is $\tau_{V}$-open. Namely, $M \in\langle\mathcal{U}\rangle \subset g^{-1}(U)$ for some finite family $\mathcal{U}$ of open subsets of $X$. Then, $M=\bigcup \mathcal{U}$ because $\mathcal{M}$ is maximal with respect to the inclusion. Thus, $M$ is a clopen subset of $X$ which contains $F$ because $F \in \mathcal{M}$. Finally note that $g(M) \in U$ which implies $x \notin M$. Hence, $G=X \backslash M$ is a clopen neighbourhood of $x$ with $G \subset V$. This completes the proof of Theorem 1.4.

\section{REFERENCES}

1. G. Artico, U. Marconi, R. Moresco and J. Pelant, Selectors and Scattered Spaces, Topology Appl., to appear.

2. D. Bertacchi and C. Costantini, Existence of selections and disconnectedness properties for the hyperspace of an ultrametric space, Topology Appl. 88 (1998), 179-197. MR 99g:54003

3. M. Choban, Many-valued mappings and Borel sets. I, Trans. Moscow Math. Soc. 22 (1970), 258-280.

4. C. Costantini and V. Gutev, Recognizing special metrics by topological properties of the "metric"-Proximal hyperspace, preprint.

5. R. Engelking, R. W. Heath, and E. Michael, Topological well-ordering and continuous selections, Invent. Math. 6 (1968), 150-158. MR 39:6272

6. V. Gutev and T. Nogura, Selections for Vietoris-like hyperspace topologies, Proc. London Math. Soc. 80 (1) (2000), 235-256. CMP 2000:04

7. E. Michael, Topologies on spaces of subsets, Trans. Amer. Math. Soc. 71 (1951), 152-182. MR 13:54f

8. J. van Mill and E. Wattel, Selections and orderability, Proc. Amer. Math. Soc. 83 (1981), 601-605. MR 82i:54038

9. T. Nogura and D. Shakhmatov, Characterizations of intervals via continuous selections, Rendiconti del Circolo Matematico di Palermo, Serie II 56 (1997), 317-328. MR 99d:54012 
10. T. Nogura and D. Shakhmatov, Spaces which have finitely many continuous selections, Bollettino U. M. I. (7) 11-A (1997), 723-729. MR 99a:54011

11. T. C. Przymusiński, On the dimension of product spaces and an example of M. Wage, Proc. Amer. Math. Soc. 76 (1979), 315-321. MR 80f:54033

12. M. L. Wage, The dimension of product spaces, preprint (1977).

13. M. L. Wage, The dimension of product spaces, Proc. Mat. Acad. Sci. USA 75 (1978), 46714672. MR 80a:54064

School of Mathematical and Statistical Sciences, Faculty of Science, University of Natal, King George V Avenue, Durban 4041, South Africa

E-mail address: gutev@sci.und.ac.za

Department of Mathematics, Faculty of Science, Ehime University, Matsuyama, 790 JAPAN

E-mail address: nogura@ehimegw.dpc.ehime-u.ac.jp 